\title{
Ab initio modelling of steady rotating stars
}

Michel Rieutord and Francisco Espinosa Lara

\begin{abstract}
Modelling isolated rotating stars at any rotation rate is a challenge for the next generation of stellar models. These models will couple dynamical aspects of rotating stars, like angular momentum and chemicals transport, with classical chemical evolution, gravitational contraction or mass-loss. Such modelling needs to be achieved in two dimensions, combining the calculation of the structure of the star, its mean flows and the time-evolution of the whole. We present here a first step in this challenging programme. It leads to the first self-consistent two-dimensional models of rotating stars in a steady state generated by the ESTER code. In these models the structure (pressure, density and temperature) and the flow fields are computed in a self-consistent way allowing the prediction of the differential rotation and the associated meridian circulation of the stars. After a presentation of the physical properties of such models and the numerical methods at work, we give the first grid of such models describing massive and intermediate-mass stars for a selection of rotation rates up to $90 \%$ of the breakup angular velocity.
\end{abstract}

Michel Rieutord

Université de Toulouse; UPS-OMP; IRAP; Toulouse, France and CNRS; IRAP; 14 avenue

E. Belin, 31400 Toulouse, France e-mail: Michel.Rieutord@irap.omp.eu

Francisco Espinosa Lara

Université de Toulouse; UPS-OMP; IRAP; Toulouse, France and CNRS; IRAP; 14 avenue

E. Belin, 31400 Toulouse, France e-mail: Francisco.Espinosa@irap.omp.eu 


\section{Introduction}

\subsection{The astrophysical context}

In the last ten years rotation has become an essential part of stellar models. It influences all stages of stellar evolution. For instance, during the formation process, a drastic reduction of the specific angular momentum, by a factor of order $10^{5}$, is observed when matter passes from the initial molecular cloud to the newly-born main-sequence star. Later, during the evolution of the star on the main sequence, many hydrodynamical instabilities, along with meridian circulations, drive some mixing in the radiative zones. The effects of this mixing are actually observed on the surface of many stars, which show elements obviously synthesized in their core region. In addition, recent work by Frischknecht et al. (2012) shows that rotation plays a role in the nucleosynthesis of s-elements in massive stars. Effects of rotation are also thought to be important in understanding the evolution and yields of the first generation of stars. Lacking in metals, these stars were more compact than present stars and had also weaker winds. Thus, their rotation was certainly faster than that of present day stars. It is therefore crucial to master rotational effects in order to reconstruct the history of metal enrichment in galaxies and to understand how the observed stars have been influenced by the first generation. Lastly, we may mention an example of the importance of rotation in the end of the life of stars: recent works on the gravitational collapse of massive stars (e.g. Metzger et al. 2011) insist on the importance of the combined effect of rotation and magnetic fields to model the explosion of supernovae and the associated production of a gamma-ray burst. The few foregoing examples are just illustrative, since rotation influences many other aspects of stellar physics like the oscillation spectrum, mass-loss etc.

\subsection{The 1D-models}

Presently, rotation is included in spherically symmetric stellar models through the approach proposed by Zahn (1992). Since these models are one-dimensional all of the effects of rotation are averaged on spheres. Thus differential rotation is only in the radial direction (said to be shellular) and no meridian flow appears explicitly. Because only the first terms of the spherical harmonic expansion of fields are included, this modelling is valid at small rotation rates. An equally important part of the models is that they assume the existence of some small-scale turbulence in the radiative regions that efficiently transport momentum horizontally (i.e. on isobars), erasing latitudinal gradients of angular velocity. 
Although limited to slow rotation and needing some ad hoc constants (like turbulent diffusivities), this approach has the great merit of allowing the use of 1D-models, which are now very precise as far as microphysics is concerned. It thus allowed investigators to reproduce many observed features of stars: surface abundance of lithium as a function mass (e.g. Charbonnel \& Talon 1999), the (relative) high number of red super-giants in low-metallicity galaxies (e.g. Maeder \& Mevnet 2001), or the ratio of type Ibc to type II supernovae (e.g. Mevnet \& Maeder 2005), etc.

Although these examples reflect a truly successful modelling, the understanding of the effects of rotation is still incomplete because in many circumstances rotation is fast. Stellar conditions thus do not meet the requirements of the models and the use of Zahn's approach becomes dubious. Clearly, we are missing a more detailed view of reality. For instance, no one knows the rotation rates that are allowed in the foregoing $1 \mathrm{D}$ models.

\subsection{The history of $2 D$-models of rotating stars}

The solution to the deficiencies of 1D-models about rotation will come from the use of multi-dimensional models (two-dimensional at least), which in-

clude the mean-field hydrodynamics along with the centrifugal distortion of the star. Unfortunately, building such models turned out to be a very difficult challenge. The story of this modelling is illuminating and we briefly summarize it below, following the more detailed account of Rieutord (2006b).

The first step dates back to polytropic hydrostatic models of James (1964). An important step forward was made by a US group around P. Bodenheimer and J. Ostriker (Ostriker \& Mark 1968; Ostriker \& Bodenheimer 1968; Mark 1968; Ostriker \& Hartwick 1968; Jackson 1970; Bodenheimer \& Ostriker 1970; Bodenheimer 1971; Bodenheimer \& Ostriker 1973). Their works are based on the Self-Consistent Field method which solves Poisson's equation for the gravitational potential, $\Delta \phi=4 \pi G \rho$, with the Green function, namely

$$
\phi(\mathbf{x})=-G \int \frac{\rho\left(\mathbf{x}^{\prime}\right)}{\left|\mathbf{x}-\mathbf{x}^{\prime}\right|} d^{3} \mathbf{x}^{\prime} .
$$

This solution readily includes the boundary conditions at infinity for the gravitational field.

Making general stellar models was impeded by many numerical difficulties. The codes could not deal with stellar masses less than $9 \mathrm{M}_{\odot}$ nor with very rapid rotation. An indication of the precision reached by these models is given by the virial test (see below). Jackson (1970) found it to be around $4 \times 10^{-3}$.

Soon after, M. Clement took up the challenge and solved directly the Poisson equation with a finite difference discretization (Clement 1974). Results improved as testified by a better virial test at $2 \times 10^{-4}$. 
Another series of work aimed at the construction of 2D-models was published by the Japanese school led by Y. Eriguchi (see Eriguchi 1978; Eriguchi \& Sugimoto 1981). These works led to the first attempts to account for the baroclinicity of radiative zones (Uryu \& Eriguchi 1994, 1995), but the neglecting of viscosity imposed the prescription of some ad hoc constraints. The series ended with the work of Shindo et al. (1997) who improved the microphysics but relaxed the dynamics, considering only barotropic models.

To be complete, we need to mention the work of Eriguchi \& Müller (1985, 1991) who attacked the problem in a different way, using a mapping of the star. The grid points are indeed mapped to the sphere through the relation

$$
r_{i}\left(\theta_{k}\right)=\zeta_{i} R_{s}\left(\theta_{k}\right)
$$

where $\zeta$ is a new radial variable spanning $[0,1]$. Finite differences are used for both variables $\zeta$ and $\theta$. The ensuing code was quite robust, being able to compute configurations quite far from the pure sphere, but the precision of calculation as monitored by the virial test remained around $5 \times 10^{-4}$.

The more recent efforts on 2D modelling not related to the ESTER project have been those of Roxburgh (2004), motivated by the need of models of rapidly rotating stars for asteroseismology, and those of Jackson et al. $(2004,2005)$ motivated by the discovery of the extreme flattening of the Be star Achernar (e.g. Domiciano de Souza et al. 2005). These two works use barotropic models with an imposed rotation law. We note that Jackson et al. (2004) used a new version of the Self-Consistent Field technique which leads to a much more robust code, not restricted to a given mass range. These models have also been used to investigate the acoustic oscillation spectrum of rapidly rotating stars by (Reese et al. 2009b,a). Lastly, Deupree (2011) worked out a series of $2 \mathrm{D}$-models with uniform rotation for masses between 1.625 and $8 \mathrm{M}_{\odot}$.

\section{The route to ideal models}

As may be observed, the weakest point of the foregoing models is their lack of dynamics as well as of time-evolution. We recall that any radiative region of a rotating star is pervaded by baroclinic flows that appear in the first place as a differential rotation and a meridian circulation (e.g. Rieutord 2006a). These flows control some turbulence there and affect the whole star, playing an important role in the transport of elements and momentum.

We may now wonder what would be the ideal model of an isolated rapidly rotating star. This question was addressed in Rieutord (2006b) and we reproduce here his discussion, which is still fully relevant:

"Such a model should describe the mean state of a star at any time of its life and especially the new quantity specific to these stars: angular momentum. 
"Unlike a non-rotating star, which is a one-dimensional object (in a largescale description) which needs only scalar fields ([we] forget magnetic fields), a rotating star is, at least, a two-dimensional object with, at least, one vector field in addition to all scalar fields. Hence, complexity increases not only by the multi-dimensional nature of the model but also by the number of physical quantities to be determined. This implies that the ideal model deals consistently with angular momentum and especially the losses and gains through stellar winds and accretion. Such a model should also take into account the baroclinicity of radiative zones and there, the anisotropic turbulence which appears through shear instabilities; it should also include a mean-field theory of convection to forecast Reynolds stresses and heat flux. Of course, observers would like to know the emissivity of the atmosphere as a function of latitude (if they use interferometry) or as a function of wavelength if they do spectroscopy. But if they do asteroseismology they surely wish to know the eigenspectrum of these objects.

"The foregoing points show that progress in the understanding of rotating stars needs also some advances in the following questions of stellar physics:

- How angular momentum is distributed in a star and how is it input or output with what consequences?

- The immediately following question concerns the nature of the Reynolds stresses in the convective and radiative zones.

- Then, what is the baroclinic state of the radiative regions ?

- Similarly, the atmosphere is in a baroclinic state and cannot be at rest: how strong are the differential rotation and the meridional currents? Does the atmosphere develop strong azimuthal winds streaming around the star like Jupiter's winds?

- Gravity darkening can be so efficient that equatorial regions are cool enough to develop convection; this raises the question of the latitude dependence of emissivity of the atmosphere beyond the von Zeipel model (see the attempt of Lovekin et al. 2006).

- [we] did not mention magnetic fields. Clearly, they multiply the number of problems and first steps should ignore them if possible."

\section{Setting the problem}

Many of the foregoing questions can be answered by steady rotating stars, which do not evolve, neither dynamically by losing mass and angular momentum, nor by gravitationally contracting, nor by nuclear evolution. As a first step towards the general models, we concentrate on this simpler problem. 


\subsection{Equations for a steady rotating star}

We consider a lonely rotating star in a steady state. The star is governed by the following equations for macroscopic quantities:

$$
\left\{\begin{array}{l}
\Delta \phi=4 \pi G \rho \\
\rho T \mathbf{v} \cdot \nabla s=-\operatorname{div} \mathbf{F}+\varepsilon_{*} \\
\rho\left(2 \boldsymbol{\Omega}_{*} \wedge \mathbf{v}+\mathbf{v} \cdot \boldsymbol{\nabla} \mathbf{v}\right)=-\nabla P-\rho \boldsymbol{\nabla}\left(\phi-\frac{1}{2} \Omega_{*}^{2} s^{2}\right)+\mathbf{F}_{v} \\
\operatorname{div}(\rho \mathbf{v})=0
\end{array}\right.
$$

The first equation is Poisson's equation which relates the mass distribution ( $\rho$ is the density) and the gravitational potential $\phi$ ( $G$ is the gravitation constant). The second equation is the heat equation, which relates the advection of entropy $s$ by the velocity field $\mathbf{v}$ when nuclear heat sources $\varepsilon_{*}$ are present, with the heat flux $\mathbf{F}$. The third equation is the momentum equation written in a frame rotating at angular velocity $\boldsymbol{\Omega}_{*} . P$ is the pressure field and $\mathbf{F}_{v}$ the viscous force. Finally the last equation ensures mass conservation.

These equations need to be completed by those describing the microphysics and the transport properties of stellar matter. We propose to describe the heat flux with

$$
\mathbf{F}=-\chi_{r} \nabla T-\frac{\chi_{\mathrm{turb}} T}{\mathcal{R}_{M}} \nabla s
$$

where $\chi_{r}$ is the radiative conductivity and $\chi_{\text {turb }}$ a turbulent heat conductivity. In this expression the second term is assumed to represent the convective heat flux, which is supposed to be controlled by the entropy gradient. $\mathcal{R}_{M}=\mathcal{R} / \mathcal{M}$ where $\mathcal{R}$ is the ideal gas constant and $\mathcal{M}$ the mean molecular mass of the fluid.

A realistic modelling of the viscous force would be derived from the turbulent Reynolds stresses, however before reaching this long term goal we assume the simple model of a constant dynamical viscosity $\mu$, namely:

$$
\mathbf{F}_{v}=\mu\left(\Delta \mathbf{v}+\frac{1}{3} \nabla \operatorname{div} \mathbf{v}\right)
$$

The microphysics is completed by the three expressions

$$
\left\{\begin{array}{l}
P \equiv P(\rho, T) \\
\kappa \equiv \kappa(\rho, T) \\
\varepsilon_{*} \equiv \varepsilon_{*}(\rho, T)
\end{array}\right.
$$

which respectively give the equation of state, the opacities and the nuclear heat generation. We recall that in radiative diffusive equilibrium, heat conductivity is related to opacity by 


$$
\chi_{r}=\frac{16 \sigma T^{3}}{3 \kappa \rho},
$$

where $\sigma$ is the Stefan-Boltzmann constant.

\subsection{Boundary conditions - Angular momentum condition}

The previous system of partial differential equations needs to be completed by boundary conditions which affect the gravitational potential, the velocity field, the pressure and temperature.

At the star centre these conditions are simply that all functions should be regular. At the surface things are more difficult. First because the surface of a star is ill-defined. To simplify, we shall assume that the surface is an isobar or an equipotential. If the properties of the model are independent of the chosen surface, then we may say that the choice of the bounding surface is not crucial. On this surface we have to:

- match the gravitational potential to that in the vacuum, which vanishes at infinity;

- impose stress-free conditions on the velocity field, namely that

$$
\mathbf{v} \cdot \mathbf{n}=0 \quad \text { and } \quad([\sigma] \mathbf{n}) \wedge \mathbf{n}=\mathbf{0}
$$

where $[\sigma]$ is the stress tensor. The fluid is assumed to not flow outside the star, and to feel no horizontal stress ( $\mathbf{n}$ is the outer normal of the star);

- specify the pressure on the surface. A simple choice is $P_{s}=\frac{2}{3} \frac{\bar{g}}{\bar{\kappa}}$, where $\bar{\kappa}$ and $\bar{g}$ are the averaged opacity and gravity on this surface;

- impose the temperature boundary conditions. To simplify we assume that the star radiates locally as a black body. Therefore we ask

$$
\mathbf{n} \cdot \nabla T+T / L_{T}=0
$$

where $L_{T}$ is specifying the scale of temperature variations near the surface. We note that if the diffusion approximation is used then $L_{T}=$ $16 /(3 \rho \kappa), \kappa$ being the opacity.

We note that this problem, as formulated by Eqs. (1), is not fully constrained because the total angular momentum is not specified. We have specified the rotation rate of the frame where the solution is computed but this is not specifying the rotation rate of matter, which is the combination of both $\mathbf{v}$ and the solid body rotation of the frame. To remove this degeneracy we may either specify the total angular momentum of the matter or specify the equatorial velocity of the star. In the first case we may write 


$$
\int_{(V)} r \sin \theta \rho u_{\varphi} d V=0
$$

and

$$
v_{\varphi}(r=R, \theta=\pi / 2)=0
$$

in the second case. The first condition just specifies that all the angular momentum of the star is in the solid body rotation of the frame, while the second just says that the frame is rotating at the equatorial angular velocity.

\subsection{Scalings}

As formulated by (11), the problem is that of the steady flow of a selfgravitating compressible gas subject to some heat sources. As all fluid mechanics problem we first need to choose the various relevant scales that feature the physical quantities. These scales are used to rewrite the set of equations with dimensionless variables. We converged to the following set:

Length scale: equatorial radius $\ldots \ldots \ldots \ldots \ldots \ldots \ldots \ldots \ldots$

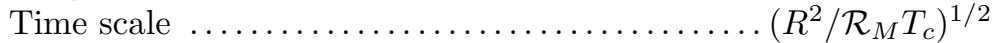

Velocity scale $\ldots \ldots \ldots \ldots \ldots \ldots \ldots \ldots \ldots \ldots . . \ldots+\sqrt{\mathcal{R}_{M} T_{c}}$

Density scale: central density $\ldots \ldots \ldots \ldots \ldots \ldots \ldots \ldots \ldots \rho_{c}$

Temperature scale: central temperature $\ldots \ldots \ldots \ldots \ldots \ldots \ldots T_{c}$

Pressure scale $\ldots \ldots \ldots \ldots \ldots \ldots \ldots \ldots \ldots \ldots \ldots \ldots \ldots \mathcal{R}_{M} \rho_{c} T_{c}$

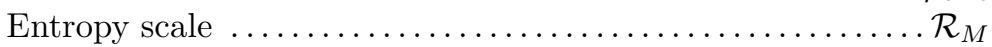

Potential scale $\ldots \ldots \ldots \ldots \ldots \ldots \ldots \ldots \ldots \ldots \ldots . \ldots \ldots R^{2} \rho_{c}$

This scaling uses the sound travel time as the time scale, and as a consequence the velocity field is scaled by a central sound velocity. Accordingly, the potential scale would be $V^{2}$, but we prefer to use $4 \pi G R^{2} \rho_{c}$, which comes from the free-fall time scale.

\subsection{Dimensionless equations and numbers}

Using the foregoing scaling leads to the following dimensionless equations

$$
\left\{\begin{array}{l}
\rho\left(2 \Omega \mathbf{e}_{z} \wedge \mathbf{u}+(\mathbf{u} \cdot \boldsymbol{\nabla}) \mathbf{u}\right)=-\boldsymbol{\nabla} p-\rho \boldsymbol{\nabla}\left(\Lambda_{p} \psi-\frac{1}{2} \Omega^{2} s^{2}\right) \\
\quad+E\left(\Delta \mathbf{u}+\frac{1}{3} \boldsymbol{\nabla} \operatorname{div} \mathbf{u}\right) \\
\rho T \mathbf{u} \cdot \boldsymbol{\nabla} s=\operatorname{div}\left(\chi \nabla T+\chi_{t} T \nabla s\right)+\varepsilon \\
\operatorname{div} \rho \mathbf{u}=0 \\
\Delta \psi=\rho
\end{array}\right.
$$


with the numbers

$$
\Omega=\frac{\Omega_{*} R}{\sqrt{\mathcal{R}_{M} T_{c}}}, \quad E=\frac{\mu}{\rho_{c} R \sqrt{\mathcal{R}_{M} T_{c}}}, \quad \Lambda_{p}=\frac{4 \pi G R^{2} \rho_{c}}{\mathcal{R}_{M} T_{c}}
$$

and the dimensionless functions

$$
\psi=\frac{\phi_{*}}{4 \pi G R^{2} \rho_{c}}, \quad \chi=\frac{\chi_{r}(\rho, T)}{\mathcal{R}_{M} \rho_{c} R \sqrt{\mathcal{R}_{M} T_{c}}}, \quad \varepsilon=\frac{R \varepsilon_{*}(\rho, T)}{\rho_{c}\left(\mathcal{R}_{M} T_{c}\right)^{3 / 2}} .
$$

Although the critical angular velocity can only be computed after the completion of the calculation, it is useful to define the pseudo-critical angular velocity $\Omega_{c}=\sqrt{4 \pi G \rho_{c}}$,

$$
\frac{\Omega_{*}}{\Omega_{c}}=\frac{\Omega}{\sqrt{\Lambda_{p}}}
$$

and introduce other dimensionless functions, namely

$$
\bar{\varepsilon}=\varepsilon / \varepsilon(0), \quad \bar{\chi}=\chi / \chi(0), \quad \Lambda_{T}=\frac{\varepsilon(0)}{\chi(0)}=\frac{R^{2} \varepsilon_{*}\left(\rho_{c}, T_{c}\right)}{T_{c} \chi_{r}\left(\rho_{c}, T_{c}\right)} .
$$

\subsection{Global parameters, $\rho_{c}, T_{c}, R$ of the star model}

Let us suppose that we know the mass of the star $M$, how can the foregoing equations be used to determine the stellar quantities, especially $\rho_{c}, T_{c}$ and $R ?$

If we have solved the problem, we observe that $\Lambda_{p}$ is known, which gives a first relation between $\rho_{c}, T_{c}$ and $R^{2}$. In the same manner, the central value of $\varepsilon$ gives a relation between $\rho_{c}$ and $T_{c}$ :

$$
\varepsilon(0)=\frac{R \varepsilon_{*}\left(\rho_{c}, T_{c}\right)}{\rho_{c}\left(\mathcal{R}_{M} T_{c}\right)^{3 / 2}} .
$$

Thus, taking into account that the mass of the star expresses as a function of $R$ and $\rho_{c}$, namely

$$
M=\rho_{c} R^{3} \int_{(V)} \rho d V
$$

the expressions of $\Lambda_{p}$ and $\varepsilon(0)$ completely determine the parameters of the stars, i.e. the radius, the central temperature and central density. 


\subsection{Numerical method}

The resolution of the set of equations (3) imposes some numerical challenges. First, the shape of a rotating star is spheroidal and not known a priori. We thus use a mapping of coordinates adapted to this geometry and modify it during the calculation. Secondly, as the problem is two-dimensional, its size quickly grows with the resolution, thus imposing the use of an efficient numerical method that should be, at the same time, precise enough to deal with velocity fields. Finally, as the problem is non-linear, we solve it using an iterative procedure.

\subsubsection{Computational domain and mapping of coordinates}

Following Bonazzola et al. (1998), we use a mapping of the coordinates $r(\zeta, \theta)$ adapted to the geometry of the star. The new spheroidal coordinates $(\zeta, \theta)$, are defined in such a way that $\zeta=1$ represents the surface of the star, $\theta$ being the colatitude. Thus doing, the surface of the star is a surface of coordinate, a property that very much simplifies the implementation of boundary conditions.

The star can be subdivided in several domains, and in each domain the relation between spherical and spheroidal coordinates is given by the general expression

$$
r=a_{i} \zeta+A_{i}(\zeta)\left[R_{i+1}(\theta)-a_{i} \eta_{i+1}\right]+B_{i}(\zeta)\left[R_{i}(\theta)-a_{i} \eta_{i}\right] \quad \eta_{i} \leq \zeta \leq \eta_{i+1},
$$

where $B_{i}(\zeta)=1-A_{i}(\zeta)$. Here $R_{i}(\theta)$ and $R_{i+1}(\theta)$ represent the internal and external boundary of the domain respectively, and $a_{i}, A_{i}(\zeta)$ are chosen to satisfy some properties at the boundaries between the different domains. In particular, we want

$$
r\left(\zeta=\eta_{i}, \theta\right)=R_{i}(\theta) \quad \text { and } \quad r\left(\zeta=\eta_{i+1}, \theta\right)=R_{i+1}(\theta),
$$

then it should be that $A_{i}\left(\eta_{i}\right)=0$ and $A_{i}\left(\eta_{i+1}\right)=1$. The value of the parameters $a_{i}$ should be such that $r$ is monotonically increasing with $\zeta$.

A nice property of this mapping is that it reduces to the spherical coordinates near the centre. The use of a spherical harmonic expansion of the unknowns is therefore possible, and the asymptotic properties of the solutions near the centre are well-known. The decomposition of the stellar domain into multi-subdomains improves the efficiency of the spectral methods to be used, especially in dealing with discontinuities (interface between a convective and radiative region) and in dealing with the large pressure and density variations.

As we have already mentioned, at the stellar surface, the gravitational potential must match the vacuum solution vanishing at infinity. There is no easy way of imposing this condition on a surface with arbitrary shape. 


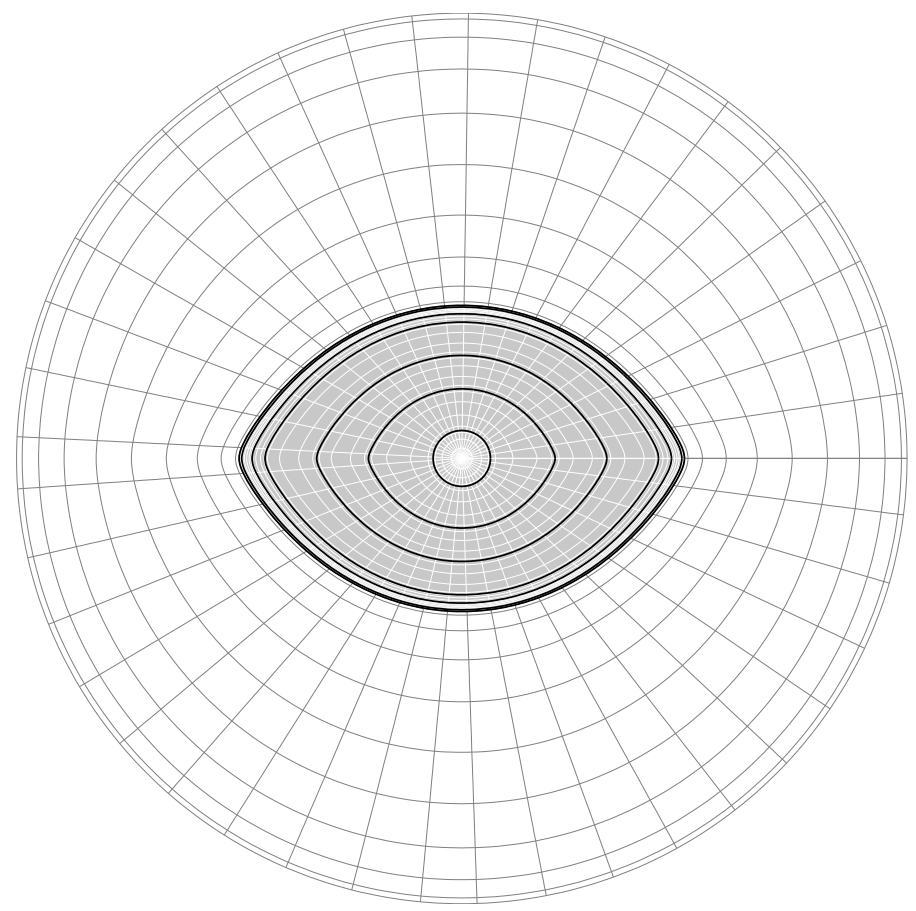

Fig. 1 The computational domain: the star at the centre is divided into sub-domains (layers) that ease the computations. It is surrounded by a vacuum domain limited by a sphere which allows an easy implementation of the boundary conditions on the gravitational potential.

To circumvent this difficulty, we follow Bonazzola et al. (1998) who use an additional domain such that $1 \leq \zeta \leq 2$ and whose outer boundary is a sphere as shown in Fig. 1. On this sphere bounding the computational domain, the gravitational potential can meet simple boundary conditions for each of its spherical harmonics, namely

$$
\frac{\partial \phi_{\ell}}{\partial r}+\frac{(\ell+1) \phi_{\ell}}{r}=0
$$

which ensure the matching with a field vanishing at infinity. Fig. 1 shows the full computational domain along with the key surfaces of the mapping.

To write down the equations (3), the operators should be written with the spheroidal coordinates. Let first us recall that the relation between these coordinates and the classical spherical coordinates is

$$
r \equiv r\left(\zeta, \theta^{\prime}\right), \quad \theta=\theta^{\prime}, \quad \varphi=\varphi^{\prime} .
$$


In order to manipulate these new coordinates, and especially the vectors, we use the natural basis defined by

$$
\mathbf{E}_{\zeta}=\frac{\partial \mathbf{r}}{\partial \zeta}, \quad \mathbf{E}_{\theta}=\frac{\partial \mathbf{r}}{\partial \theta}, \quad \mathbf{E}_{\varphi}=\frac{\partial \mathbf{r}}{\partial \varphi}
$$

Making explicit these definitions, we can express the covariant and contravariant vectors, namely:

$$
\begin{aligned}
\mathbf{E}_{\zeta} & =r_{\zeta} \mathbf{e}_{r}, \quad \mathbf{E}_{\theta}=r_{\theta} \mathbf{e}_{r}+r \mathbf{e}_{\theta}, \quad \mathbf{E}_{\varphi}=r \sin \theta \mathbf{e}_{\varphi} \\
\mathbf{E}^{\zeta} & =\frac{\mathbf{e}_{r}}{r_{\zeta}}-\frac{r_{\theta}}{r r_{\zeta}} \mathbf{e}_{\theta}, \quad \mathbf{E}^{\theta}=\frac{\mathbf{e}_{\theta}}{r}, \quad \mathbf{E}^{\varphi}=\frac{\mathbf{e}_{\varphi}}{r \sin \theta}
\end{aligned}
$$

as functions of the usual unit vectors associated with spherical coordinates $\left(\mathbf{e}_{r}, \mathbf{e}_{\theta}, \mathbf{e}_{\varphi}\right)$; we set:

$$
r_{\zeta}=\frac{\partial r}{\partial \zeta}, \quad r_{\theta}=\frac{\partial r}{\partial \theta} .
$$

Passing from the spherical to the spheroidal coordinates may be done using the following relations:

$$
\frac{\partial f}{\partial r}=\frac{1}{r_{\zeta}} \frac{\partial f}{\partial \zeta}, \quad \frac{\partial f}{\partial \theta}=\frac{\partial f}{\partial \theta^{\prime}}-\frac{r_{\theta}}{r_{\zeta}} \frac{\partial f}{\partial \zeta}, \quad \frac{\partial f}{\partial \varphi}=\frac{\partial f}{\partial \varphi^{\prime}} .
$$

These expressions give the metric tensor whose covariant $\left(g_{i j}=\mathbf{E}_{i} \cdot \mathbf{E}_{j}\right)$ and contravariant $\left(g^{i j}=\mathbf{E}^{i} \cdot \mathbf{E}^{j}\right)$ components are:

$$
\begin{gathered}
g_{\zeta \zeta}=r_{\zeta}^{2}, \quad g_{\zeta \theta}=r_{\zeta} r_{\theta}, \quad g_{\theta \theta}=r^{2}+r_{\theta}^{2}, \quad g_{\varphi \varphi}=r^{2} \sin ^{2} \theta \\
g^{\zeta \zeta}=\frac{r^{2}+r_{\theta}^{2}}{r^{2} r_{\zeta}^{2}}, \quad g^{\zeta \theta}=-\frac{r_{\theta}}{r^{2} r_{\zeta}}, \quad g^{\theta \theta}=\frac{1}{r^{2}}, \quad g^{\varphi \varphi}=\frac{1}{r^{2} \sin ^{2} \theta}
\end{gathered}
$$

and $g_{\zeta \varphi}=g_{\theta \varphi}=g^{\zeta \varphi}=g^{\theta \varphi}=0$. At $r=0, g^{\theta \theta}, g^{\varphi \varphi}$ are singular. The metric determinant is such that

$$
\sqrt{|g|}=r^{2} r_{\zeta} \sin \theta \quad \text { and } \quad\left|\varepsilon^{i j k}\right|=\frac{1}{\sqrt{|g|}}
$$

The volume element is given by

$$
d V=\sqrt{|g|} d \zeta d \theta d \varphi=r^{2} r_{\zeta} \sin \theta d \zeta d \theta d \varphi=r^{2} r_{\zeta} d \zeta d \Omega .
$$

As an example, the Poisson equation for the gravitational potential takes the form 


$$
\begin{aligned}
& \qquad \begin{array}{c}
\Delta \psi=g^{\zeta \zeta} \frac{\partial^{2} \psi}{\partial \zeta^{2}}+\left[\frac{2}{r_{\zeta}}\left(1+\frac{r_{\theta} r_{\zeta \theta}}{r r_{\zeta}}\right)-g^{\zeta \zeta} \frac{r_{\zeta \zeta}}{r_{\zeta}}-\frac{r_{\theta \theta}+r_{\theta} \cot \theta}{r^{2} r_{\zeta}}\right] \frac{\partial \psi}{\partial \zeta}- \\
\quad-\frac{2 r_{\theta}}{r^{2} r_{\zeta}} \frac{\partial^{2} \psi}{\partial \zeta \partial \theta}+\frac{1}{r^{2}}\left[\frac{1}{\sin \theta} \frac{\partial}{\partial \theta}\left(\sin \theta \frac{\partial \psi}{\partial \theta}\right)+\frac{1}{\sin ^{2} \theta} \frac{\partial^{2} \psi}{\partial \varphi^{2}}\right] \\
=\rho,
\end{array} \\
& \text { where } r_{\zeta \zeta}=\frac{\partial^{2} r}{\partial \zeta^{2}}, r_{\theta \theta}=\frac{\partial^{2} r}{\partial \theta^{2}} \text { and } r_{\zeta \theta}=\frac{\partial^{2} r}{\partial \zeta \partial \theta} .
\end{aligned}
$$

\subsubsection{Numerical method}

As we have mentioned earlier, due to the 2D nature of the problem, the size of the calculation will increase very quickly with the number of grid points. This motivates the use of a high order method that can achieve high precision with low resolution grids, optimizing computation time and memory requirements.

An additional difficulty comes from the fact that the overall structure of the star (profile of pressure, temperature, etc.) and its dynamics (rotation, meridional circulation) must be computed simultaneously. This requires a great precision and the ability to calculate higher order derivatives of some variables.

Spectral methods are specially well-suited for this kind of problem Grandclément 2006; Canuto et al. 2006). These methods expand the solutions on a basis of orthogonal functions. The approximation of the solution using $n$ basis functions will be

$$
\phi^{(n)}(x)=\sum_{l=0}^{n-1} a_{l} P_{l}(x) .
$$

The basis functions $P_{l}(x)$ used are usually a set of orthogonal polynomials, such as the Legendre or Chebyshev polynomials. In our case it can be shown that if $\phi(x)$ is infinitely differentiable, the approximate function $\phi^{(n)}(x)$ will converge to the exact solution $\phi(x)$ faster than any power of the grid resolution $h$. This expresses the fact that, in spectral methods, the error decreases exponentially with the number of basis functions $n$ (Fornberg 1998). The $i$-th derivative of a function is approximated by

$$
\left(\frac{\mathrm{d}^{i} \phi}{\mathrm{d} x^{i}}\right)^{(n)}=\sum_{l=0}^{n-1} a_{l} \frac{\mathrm{d}^{i} P_{l}}{\mathrm{~d} x^{i}} .
$$

Due to the non-linearity of the equations that we have to solve, we have to calculate the product of two variables of the model in an efficient way. Unfortunately, multiplication cannot be easily performed using the spectral coefficients, as it involves a convolution in the transformed space. This can be solved by using an alternate approach of spectral methods, with the same properties, called pseudospectral collocation methods. In a pseudospectral 
method the variables are not represented by their spectral coefficients, but by their values at certain points $x_{i}$ called the collocation points. Then, all the calculations can be performed in the real space.

The basis functions are orthogonal against some scalar product $\left\langle P_{l}, P_{m}\right\rangle=\delta_{l m}$. Then, we can get the spectral coefficients $a_{l}$ by

$$
a_{l}=\left\langle\phi(x), P_{l}(x)\right\rangle .
$$

The scalar product (usually a weighted integral over the interval) can be calculated using the gaussian quadrature formula associated with the family of orthogonal polynomials $P_{l}$

$$
a_{l}=\sum_{j=0}^{n-1} w_{j} P_{l}\left(x_{j}\right) \phi\left(x_{j}\right),
$$

where $x_{j}$ and $w_{j}$ are the nodes and weights of the gaussian quadrature. Note that $x_{j}$ are the collocation points. Then, the first derivative at the collocation points can be obtained as

$$
\begin{aligned}
\phi^{\prime}\left(x_{i}\right) & =\sum_{l=0}^{n-1}\left(\sum_{j=0}^{n-1} w_{j} P_{l}\left(x_{j}\right) \phi\left(x_{j}\right)\right) P_{l}^{\prime}\left(x_{i}\right) \\
& =\sum_{j=0}^{n-1}\left(\sum_{l=0}^{n-1} w_{j} P_{l}\left(x_{j}\right) P_{l}^{\prime}\left(x_{i}\right)\right) \phi\left(x_{j}\right) \\
& =\sum_{j=0}^{n-1} D_{i j} \phi\left(x_{j}\right)
\end{aligned}
$$

where $D_{i j}$ is the differentiation matrix.

We use this procedure in the radial and latitudinal directions to transform the original system of non-linear partial differential equations in a system of non-linear algebraic equations. For the latitudinal direction, we use Legendre polynomials as the basis functions, while for the radial direction we use Chebyshev polynomials associated with the Gauss-Lobatto collocation points. This grid includes the points at the extrema in order to deal with boundary conditions.

The nice convergence properties of spectral and pseudospectral methods are only valid for smooth functions which are infinitely differentiable. However, it is known that inside a star there will be some discontinuities, as for example, at the boundary between a convective core and a radiative envelope. This difficulty is solved by using a multi-domain approach, in a way that the variables are continuous and differentiable within each domain but not necessarily at the boundaries between different domains. 


\subsubsection{Iterative procedure}

The system of algebraic equations resulting from the discretization of the problem is non-linear and is solved using an iterative method. For that we either use the well-known Newton's method or a relaxation method. For Newton's method we write the problem in the form

$$
\mathbf{F}(\mathbf{x})=\mathbf{0}
$$

where the vector function $\mathbf{F}$ represents the equations that we want to solve and $\mathbf{x}$ is the vector containing all the independent variables of the problem (pressure, temperature, ...) including the shape of the surface which is not

known a priori. The equations are linearized using the Jacobian matrix of $\mathbf{F}(\mathbf{x})$ defined as

$$
\delta \mathbf{F}(\mathbf{x})=\mathrm{J}(\mathbf{x}) \delta \mathbf{x} .
$$

Then the correction to the solution in the $k$-th iteration will be calculated solving the linear system

$$
\mathrm{J}\left(\mathbf{x}^{(k)}\right) \delta \mathbf{x}^{(k)}=-\delta \mathbf{F}\left(\mathbf{x}^{(k)}\right)
$$

and we set $\mathbf{x}^{(k+1)}=\mathbf{x}^{(k)}+\delta \mathbf{x}^{(k)}$.

With an appropriate initial approximation $\mathbf{x}^{(0)}$, Newton's method has quadratic convergence. In practice, a rotating stellar model can be calculated in approximately 10 iterations starting with the corresponding non-rotating model.

\subsubsection{The case of flow fields}

The computation of the flow field is certainly the most delicate part of the solution. Its computation needs to circumvent two difficulties: first the flow faces very large variations of the density (typically eight orders of magnitude) and second the low viscosity of the stellar fluid. Indeed, this latter complication implies that the flows need to be computed within the asymptotic regime of low Ekman numbers while the zero viscosity solution is degenerate for the linear part of the velocity operator (any geostrophic flow may be added to a solution see Rieutord 2006a for detailed explanations). We shall present this rather technical point in a separate work (Espinosa Lara \& Rieutord 2012).

\subsection{Tests of the results}

We checked the results with two global tests: the virial theorem and the global balance of energy. 


\subsubsection{The virial test}

Let us first present the virial test. For this, we recall that the equations of a steady flow in a rotating frame are:

$$
\begin{aligned}
2 \boldsymbol{\Omega} \wedge \rho \mathbf{u}+\rho \mathbf{u} \cdot \boldsymbol{\nabla u} & =-\rho \boldsymbol{\nabla} \phi+\rho \Omega^{2} s \mathbf{e}_{s}+\operatorname{Div}[\sigma] \\
\operatorname{div} \rho \mathbf{u} & =0
\end{aligned}
$$

with the boundary conditions on the velocity field $\mathbf{u} \cdot \mathbf{n}=0$ and $\mathbf{n} \wedge[\sigma] \mathbf{n}=\mathbf{0}$. The virial equality is obtained by integrating the scalar product of $\mathbf{r}$ with the momentum equation over the fluid's volume. Hence, we have to evaluate the following integrals:

- The $z$-component of the relative angular momentum

$$
\int_{(V)} \mathbf{r} \cdot 2 \boldsymbol{\Omega} \wedge \rho \mathbf{u} d V=-2 \boldsymbol{\Omega} \cdot \int_{(V)} \mathbf{r} \wedge \rho \mathbf{u} d V=-2 \Omega L_{z} .
$$

- The gravitational energy

$$
-\int_{(V)} \mathbf{r} \cdot \rho \boldsymbol{\nabla} \phi d V=\frac{1}{2} \int_{(V)} \rho \phi d^{3} \mathbf{r}=W .
$$

- The kinetic energy due to bulk rotation as measured by the frame rotation

$$
\int_{(V)} \mathbf{r} \cdot \rho \Omega^{2} s \mathbf{e}_{s} d V=\int_{(V)} \rho s^{2} d V=I \Omega^{2}
$$

where $I$ is the moment of inertia.

- The relative kinetic energy

$$
\int_{(V)} \mathbf{r} \cdot \rho \mathbf{u} \cdot \nabla \mathbf{u} d V=-\int_{(V)} \rho u^{2} d V=-2 T_{\mathrm{rel}}
$$

- The stress integral

$$
\int_{(V)} \mathbf{r} \cdot \operatorname{Div}[\sigma] d V=\int_{(S)} r_{i} \sigma_{i j} d S_{j}-\int_{(V)} \sigma_{i i} d V
$$

Stellar gas is assumed to be a newtonian fluid without volume viscosity, hence the stress tensor is

$$
\sigma_{i j}=\mu c_{i j}-p \delta_{i j}
$$

where $[c]$ is the shear tensor $\left(c_{i j}=\partial_{i} v_{j}+\partial_{j} v_{i}-2\left(\partial_{k} v_{k}\right) \delta_{i j} / 3\right)$, so that $\sigma_{i i}=-3 p$.

Thus the virial equality may be written 


$$
2 T_{\mathrm{rel}}+I \Omega_{*}^{2}+W+3 P+I_{s}+2 \Omega_{*} L_{z}=0
$$

or, with non-dimensional quantities

$$
2 T_{\mathrm{rel}}+I \Omega^{2}+\Lambda_{p} W+3 P+I_{s}+2 \Omega L_{z}=0
$$

where $I_{s}$ is the surface integral that appears in the stress integral. In the case of a steady configuration like the one under consideration, the surface integral is estimated as follows:

$$
I_{s}=\int_{(S)} \mu r_{i} c_{i j} d S_{j}-\int_{(S)} p \mathbf{r} \cdot d \mathbf{S}
$$

with

$$
\mathbf{n}=\mathbf{E}^{\zeta} /\left\|\mathbf{E}^{\zeta}\right\|, \quad \mathbf{r} \cdot \mathbf{n}=\frac{r}{r_{\zeta} \sqrt{g^{\zeta \zeta}}}, \quad d S=r \sqrt{r^{2}+r_{\theta}^{2}} d \Omega .
$$

Using nondimensional quantities, this leads to

$$
I_{s}=\int_{4 \pi}\left[E\left(2 \partial_{r} u_{r}-\frac{2}{3} \operatorname{div} \mathbf{u}-\frac{r_{\theta}}{r}\left(\frac{1}{r} \frac{\partial u_{r}}{\partial \theta}+\frac{\partial u_{\theta}}{\partial r}-\frac{u_{\theta}}{r}\right)\right)-p(\theta)\right] r^{3} d \Omega .
$$

\subsubsection{The energy test}

Another test of internal coherence of the solutions is provided by the energy balance between sources and losses. The integral of the entropy equation over the fluid's volume gives:

$$
\int_{(V)} \rho T \mathbf{u} \cdot \nabla s d V=\int_{(S)}\left(\chi \nabla \nabla+\chi_{t} T \nabla s\right) \cdot d \mathbf{S}+\int_{(V)} \varepsilon d V .
$$

In the case of radiative envelopes at zero Prandtl number, this equation can be simplified to

$$
\int_{(S)}(\chi \nabla T) \cdot d \mathbf{S}+\int_{(V)} \varepsilon d V=0
$$

\section{Some results}

The foregoing algorithm has been used to compute models of rotating stars in a steady state. For these models we use an analytic expression for the energy generation rate, namely:

$$
\varepsilon_{*}(\rho, T, X, Z)=\varepsilon_{0}(X, Z) \rho^{2} T^{-2 / 3} \exp \left(A / T^{1 / 3}\right)
$$




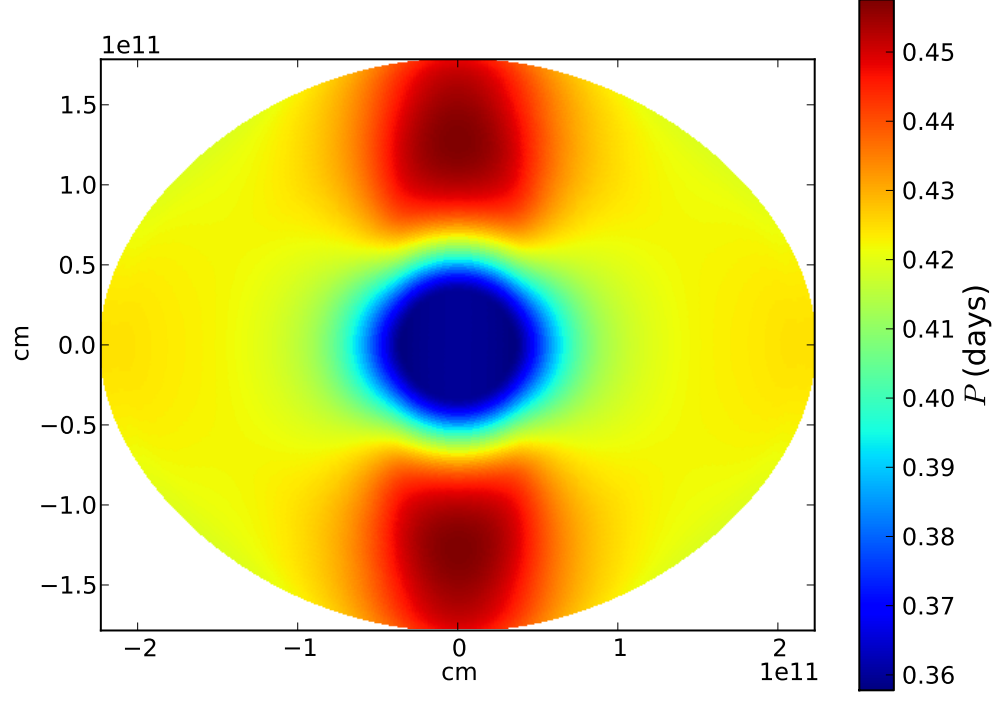

Fig. 2 Differential rotation in a $5 \mathrm{M}_{\odot}$ stellar model rotating at $\Omega=0.7 \Omega_{K}$.

as in Espinosa Lara \& Rieutord (2007). It is completed by the use of OPAL tables for the computation of the opacity and the derivation of the density from the equation of state $(X=0.7$ and $Z=0.02$ with solar composition of Grevesse \& Noels 1993).

We computed a few models of stars with a convective core, assumed to be an isentropic region, and with a radiative envelope. Presently, no convective envelope can be included in the models, which are therefore limited to stars with masses above $1.5 \mathrm{M}_{\odot}$.

Numerical difficulties come both from the high density and pressure contrast between centre and from high rotation rates. At the time of writing (April 2012) the most extreme models deal with

$$
p_{\text {surf }} / p_{\text {centre }}=10^{-14} \quad \text { and } \quad \Omega / \Omega_{K}=0.9 .
$$

The latter rotation corresponds to a flattening of 0.3 . For these models, the spatial resolution uses a spherical harmonics series truncated at $L=64$ and $N=400$ collocation points on the radial grid, which are distributed over 8 domains.

As far as the velocity field is concerned, we recall that the baroclinic flows that pervade the radiative region are computed in the asymptotic limit of vanishing Ekman numbers. Thus, the Prandtl number is also set to zero 


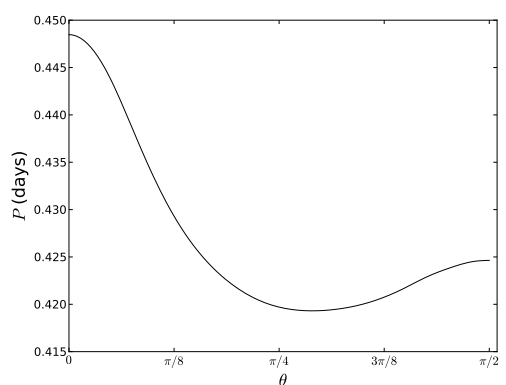

Fig. 3 The surface rotation as a function of colatitude. The stellar model is the same as that of Fig. 2

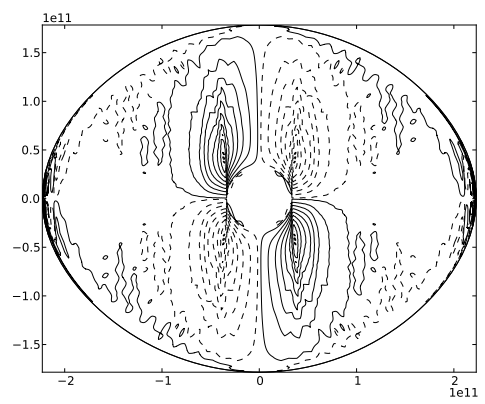

Fig. 4 Streamlines of the meridian circulation associated with the differential rotation shown in Fig. 2 Solid lines show counterclockwise circulation and dashed lines clockwise one.

and heat advection by meridional flows is neglected. Fig. 2 illustrates the differential rotation that is forced by the baroclinic torque. As also observed in previous (simpler) models of Espinosa Lara \& Rieutord (2007) and Rieutord \& Espinosa Lara (2009), the core is rotating faster than the envelope. The differential rotation is cylindrical in the isentropic core (as required by the Taylor-Proudman theorem), and almost shellular in the inner part of the radiative envelope.

The meridional circulation shown in Fig. 团is dominated by the streamlines of the Stewartson layers lying along the tangent cylinder of the convective core. This flow pattern should not be taken at face value since the interior flows are computed without viscosity. The balance of forces in the Stewartson layer cannot be ensured and therefore the flow pattern depends on the grid resolution.

In Fig. 5. we provide a view of the squared Brunt-Väisälä frequency. This shows the anisotropy of the buoyancy force which, especially in the outer layers, influences the gravito-inertial modes that are part of the oscillation spectrum of such stars (Dintrans \& Rieutord 2000).

Besides the dynamics, we have also investigated the thermal structure of rotating stars and, more specifically, the distribution of the heat flux as a function of latitude. In Espinosa Lara \& Rieutord (2011), 2D models have been used to validate a very simple model of the latitudinal variations of the flux, which depends on a single parameter $\Omega / \Omega_{K}$. Such models are based on the idea that within an envelope the heat flux $\mathbf{F}$ satisfies $\operatorname{div} \mathbf{F}=0$ and is almost anti-parallel to the local effective gravity $\mathbf{g}_{e}$. Since the mass distribution inside massive stars is concentrated the Roche model can be used, leading to latitudinal flux variations that only depend on the rotation rate. This simple model has been successfully compared with the very few observations that are available and with complete two-dimensional models (see 


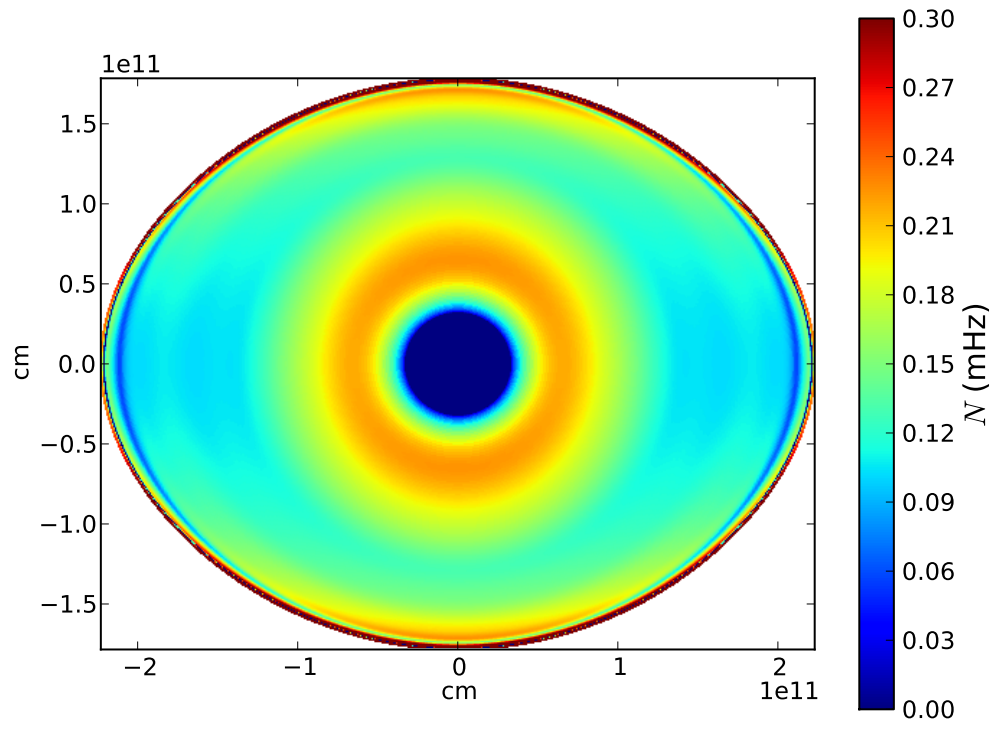

Fig. 5 The square of the Brunt-Väisälä frequency distribution in a meridian plane.

Espinosa Lara \& Rieutord 2011). As shown in Fig. 6 the two models nicely match and notably differ from the model of von Zeipel which predicts that $\mathrm{T}_{\text {eff }} \propto g_{e}^{1 / 4}$ as a consequence of neglecting of the baroclinicity of the configuration.

In Tables 1 and 2 we show the physical parameters obtained from the calculations of a series of stellar models with masses between 3 and $20 M_{\odot}$ and rotation rates up to $90 \%$ of the breakup angular velocity. The models have been calculated using $L_{\max }=64$ and 400 radial points distributed over 8 domains. The virial and energy tests give an idea of the quality of the solutions. As we can see, the virial test values are very small, below a few $10^{-10}$, as a result of the high precision of spectral methods. The energy test is also good enough (under around $10^{-5}$ ), the larger values being a consequence of the use of tabulated opacities which have limited precision.

\section{Conclusions}

The computation of two-dimensional stellar models is a real numerical challenge which has been taken up by the ESTER project. The key difficulty is to derive simultaneously the bulk structure of the star and the mean velocity 


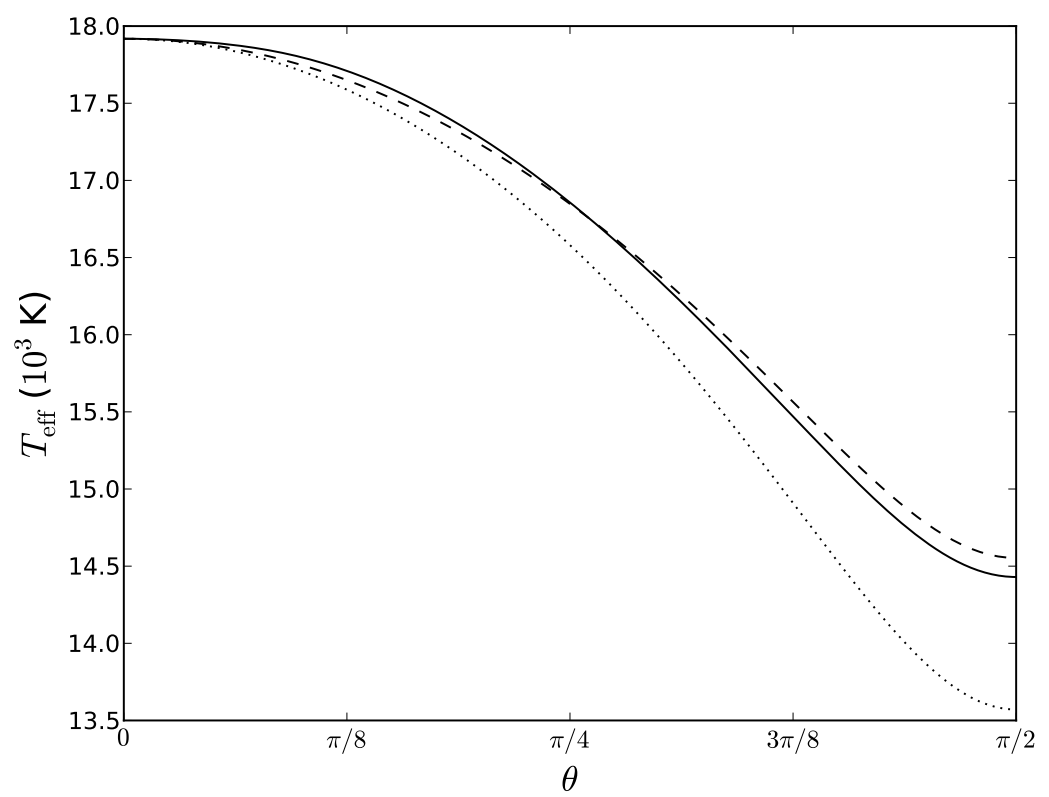

Fig. 6 Latitudinal flux variation (gravity darkening) at the surface of a rapidly rotating stellar model of $5 \mathrm{M}_{\odot}$. The solid line represents the full two-dimensional model, the dashed line the simplest model described in text and in (Espinosa Lara \& Rieutord 2011), while the dotted line shows the prediction of von Zeipel hypothesis.

fields that pervade it. These large-scale flows come from either the baroclinicity of the radiative zones or from the anisotropic Reynolds stresses in the convective zones (although some Reynolds stresses might also be expected in the radiative zones if shear instabilities can generate some small-scale turbulence as suggested by Zahn 1992). At the moment, the ESTER code can produce dynamically self-consistent models, which include the background flows but no Reynolds stresses, for stars with masses larger than $1.5 \mathrm{M}_{\odot}$ and rotation rates less than $90 \%$ the break-up. The possibilities of the code have not yet been fully explored however.

As far as steady solutions are concerned, the main challenges are to enable the modelling of the lower-mass stars (solar type) with an outer convection zone, and to take into account the effects of viscosity and Reynolds stresses in the bulk of the stars. These latter effects might indeed be crucial to the transport of elements.

The next important step will be to deal with time evolution; this should include:

- dynamical evolution during PMS phase with a slow gravitational contraction inducing spin-up of the star; 
Table 1 Fundamental parameters for a series of rotating stellar models ${ }^{a}$.

\begin{tabular}{|c|c|c|c|c|c|c|c|c|c|}
\hline $\mathrm{M}\left(\mathrm{M}_{\odot}\right)$ & $\Omega / \Omega_{K}^{b}$ & $\mathrm{R}\left(\mathrm{R}_{\odot}\right)$ & $\varepsilon^{c}$ & $P_{\mathrm{rot}}^{\mathrm{s}}(\mathrm{d})$ & $P_{\text {rot }}^{\mathrm{c}}(\mathrm{d})$ & $v_{\text {eq }}(\mathrm{km} / \mathrm{s})$ & $L\left(L_{\odot}\right)$ & $T_{\text {eff }}\left(10^{3} \mathrm{~K}\right)$ & $\log g_{e}$ \\
\hline 3.0 & 0.0 & 1.97 & 0.00 & - & - & 0.0 & 81.2 & 12.36 & 4.33 \\
\hline 3.0 & 0.3 & $\begin{array}{l}1.96(\mathrm{p}) \\
2.05(\mathrm{e})\end{array}$ & 0.04 & $\begin{array}{l}0.70(\mathrm{p}) \\
0.65(\mathrm{e})\end{array}$ & 0.54 & 158.6 & 80.0 & $\begin{array}{l}12.50(\mathrm{p}) \\
11.97(\mathrm{e})\end{array}$ & $\begin{array}{l}4.33(\mathrm{p}) \\
4.25(\mathrm{e})\end{array}$ \\
\hline 3.0 & 0.5 & $\begin{array}{l}1.95(\mathrm{p}) \\
2.19(\mathrm{e})\end{array}$ & 0.11 & $\begin{array}{l}0.46(\mathrm{p}) \\
0.43(\mathrm{e})\end{array}$ & 0.36 & 255.5 & 78.4 & $\begin{array}{l}12.69(\mathrm{p}) \\
11.31(\mathrm{e})\end{array}$ & $\begin{array}{l}4.34(\mathrm{p}) \\
4.11(\mathrm{e})\end{array}$ \\
\hline 3.0 & 0.7 & $\begin{array}{l}1.94(\mathrm{p}) \\
2.42(\mathrm{e})\end{array}$ & 0.20 & $\begin{array}{l}0.38(\mathrm{p}) \\
0.36(\mathrm{e})\end{array}$ & 0.30 & 340.4 & 77.0 & $\begin{array}{l}12.84(\mathrm{p}) \\
10.36(\mathrm{e})\end{array}$ & $\begin{array}{l}4.34(\mathrm{p}) \\
3.86(\mathrm{e})\end{array}$ \\
\hline 3.0 & 0.9 & $\begin{array}{l}1.93(\mathrm{p}) \\
2.74(\mathrm{e})\end{array}$ & 0.29 & $\begin{array}{l}0.35(\mathrm{p}) \\
0.34(\mathrm{e})\end{array}$ & 0.28 & 411.5 & 76.4 & $\begin{array}{l}12.92(\mathrm{p}) \\
8.91(\mathrm{e})\end{array}$ & $\begin{array}{l}4.34(\mathrm{p}) \\
3.32(\mathrm{e})\end{array}$ \\
\hline 5.0 & 0.0 & 2.62 & 0.00 & - & - & 0.0 & 542.8 & 17.23 & 4.30 \\
\hline 5.0 & 0.3 & $\begin{array}{l}2.60(\mathrm{p}) \\
2.72(\mathrm{e})\end{array}$ & 0.04 & $\begin{array}{l}0.83(\mathrm{p}) \\
0.77(\mathrm{e})\end{array}$ & 0.66 & 177.7 & 533.2 & $\begin{array}{l}17.44(\mathrm{p}) \\
16.69(\mathrm{e})\end{array}$ & $\begin{array}{l}4.31(\mathrm{p}) \\
4.23(\mathrm{e})\end{array}$ \\
\hline 5.0 & 0.5 & $\begin{array}{l}2.58(\mathrm{p}) \\
2.91(\mathrm{e})\end{array}$ & 0.11 & $\begin{array}{l}0.55(\mathrm{p}) \\
0.51(\mathrm{e})\end{array}$ & 0.44 & 286.5 & 520.9 & $\begin{array}{l}17.70(\mathrm{p}) \\
15.76(\mathrm{e})\end{array}$ & $\begin{array}{l}4.31(\mathrm{p}) \\
4.09(\mathrm{e})\end{array}$ \\
\hline 5.0 & 0.7 & $\begin{array}{l}2.57(\mathrm{p}) \\
3.21(\mathrm{e})\end{array}$ & 0.20 & $\begin{array}{l}0.45(\mathrm{p}) \\
0.42(\mathrm{e})\end{array}$ & 0.36 & 381.9 & 510.4 & $\begin{array}{l}17.92(\mathrm{p}) \\
14.43(\mathrm{e})\end{array}$ & $\begin{array}{l}4.32(\mathrm{p}) \\
3.83(\mathrm{e})\end{array}$ \\
\hline 5.0 & 0.9 & $\begin{array}{l}2.56(\mathrm{p}) \\
3.63(\mathrm{e})\end{array}$ & 0.30 & $\begin{array}{l}0.42(\mathrm{p}) \\
0.40(\mathrm{e})\end{array}$ & 0.33 & 461.6 & 505.3 & $\begin{array}{l}18.02(\mathrm{p}) \\
12.40(\mathrm{e})\end{array}$ & $\begin{array}{l}4.32(\mathrm{p}) \\
3.30(\mathrm{e})\end{array}$ \\
\hline 10.0 & 0.0 & 3.87 & 0.00 & - & - & 0.0 & 5733.6 & 25.55 & 4.26 \\
\hline 10.0 & 0.3 & $\begin{array}{l}3.84(\mathrm{p}) \\
4.02(\mathrm{e})\end{array}$ & 0.04 & $\begin{array}{l}1.07(\mathrm{p}) \\
0.98(\mathrm{e})\end{array}$ & 0.86 & 206.8 & 5619.0 & $\begin{array}{l}25.85(\mathrm{p}) \\
24.73(\mathrm{e})\end{array}$ & $\begin{array}{l}4.27(\mathrm{p}) \\
4.19(\mathrm{e})\end{array}$ \\
\hline 10.0 & 0.5 & $\begin{array}{l}3.81(\mathrm{p}) \\
4.29(\mathrm{e})\end{array}$ & 0.11 & $\begin{array}{l}0.70(\mathrm{p}) \\
0.65(\mathrm{e})\end{array}$ & 0.57 & 333.6 & 5469.6 & $\begin{array}{l}26.24(\mathrm{p}) \\
23.35(\mathrm{e})\end{array}$ & $\begin{array}{l}4.27(\mathrm{p}) \\
4.05(\mathrm{e})\end{array}$ \\
\hline 10.0 & 0.7 & $\begin{array}{l}3.78(\mathrm{p}) \\
4.73(\mathrm{e})\end{array}$ & 0.20 & $\begin{array}{l}0.57(\mathrm{p}) \\
0.54(\mathrm{e})\end{array}$ & 0.46 & 444.8 & 5341.5 & $\begin{array}{l}26.57(\mathrm{p}) \\
21.35(\mathrm{e})\end{array}$ & $\begin{array}{l}4.28(\mathrm{p}) \\
3.80(\mathrm{e})\end{array}$ \\
\hline 10.0 & 0.9 & $\begin{array}{l}3.76(\mathrm{p}) \\
5.37(\mathrm{e})\end{array}$ & 0.30 & $\begin{array}{l}0.53(\mathrm{p}) \\
0.51(\mathrm{e})\end{array}$ & 0.43 & 536.7 & 5279.3 & $\begin{array}{l}26.73(\mathrm{p}) \\
18.28(\mathrm{e})\end{array}$ & $\begin{array}{l}4.28(\mathrm{p}) \\
3.26(\mathrm{e})\end{array}$ \\
\hline 20.0 & 0.0 & 5.70 & 0.00 & - & - & 0.0 & 43791.2 & 35.00 & 4.23 \\
\hline 20.0 & 0.3 & $\begin{array}{l}5.66(\mathrm{p}) \\
5.91(\mathrm{e})\end{array}$ & 0.04 & $\begin{array}{l}1.36(\mathrm{p}) \\
1.24(\mathrm{e})\end{array}$ & 1.13 & 241.0 & 42921.1 & $\begin{array}{l}35.41(\mathrm{p}) \\
33.89(\mathrm{e})\end{array}$ & $\begin{array}{l}4.23(\mathrm{p}) \\
4.16(\mathrm{e})\end{array}$ \\
\hline 20.0 & 0.5 & $\begin{array}{l}5.61(\mathrm{p}) \\
6.32(\mathrm{e})\end{array}$ & 0.11 & $\begin{array}{l}0.89(\mathrm{p}) \\
0.82(\mathrm{e})\end{array}$ & 0.74 & 388.5 & 41775.4 & $\begin{array}{l}35.94(\mathrm{p}) \\
31.97(\mathrm{e})\end{array}$ & $\begin{array}{l}4.24(\mathrm{p}) \\
4.01(\mathrm{e})\end{array}$ \\
\hline 20.0 & 0.7 & $\begin{array}{l}5.57(\mathrm{p}) \\
7.00(\mathrm{e})\end{array}$ & 0.20 & $\begin{array}{l}0.72(\mathrm{p}) \\
0.68(\mathrm{e})\end{array}$ & 0.61 & 516.9 & 40790.9 & $\begin{array}{l}36.39(\mathrm{p}) \\
29.16(\mathrm{e})\end{array}$ & $\begin{array}{l}4.24(\mathrm{p}) \\
3.76(\mathrm{e})\end{array}$ \\
\hline 20.0 & 0.9 & $\begin{array}{l}5.54(\mathrm{p}) \\
8.02(\mathrm{e})\end{array}$ & 0.31 & $\begin{array}{l}0.67(\mathrm{p}) \\
0.65(\mathrm{e})\end{array}$ & 0.56 & 621.0 & 40326.9 & $\begin{array}{l}36.60(\mathrm{p}) \\
24.82(\mathrm{e})\end{array}$ & $\begin{array}{l}4.25(\mathrm{p}) \\
3.22(\mathrm{e})\end{array}$ \\
\hline \multicolumn{10}{|c|}{$\begin{array}{l}{ }^{a} \text { From left to right: Mass, equatorial angular velocity, radius, flattening, central rotation } \\
\text { period, surface rotation period, equatorial velocity, luminosity, effective temperature, } \\
\text { logarithm of effective gravity (cgs). The values for the solar parameters used in the table } \\
\text { are } M_{\odot}=1.9891 \cdot 10^{33} \mathrm{~g}, R_{\odot}=6.95508 \cdot 10^{10} \mathrm{~cm} \text { and } L_{\odot}=3.8396 \cdot 10^{33} \mathrm{erg} / \mathrm{s} \text {. } \\
{ }^{b} \Omega_{K}=\sqrt{\frac{G M}{R_{e}^{3}}}\end{array}$} \\
\hline
\end{tabular}


Table 2 Fundamental parameters for a series of rotating stellar models (cont. $)^{a}$.

\begin{tabular}{|c|c|c|c|c|c|c|c|c|}
\hline \multirow{2}{*}{$\begin{array}{l}\overline{\mathrm{M}\left(\mathrm{M}_{\odot}\right)} \\
\overline{3.0}\end{array}$} & \multicolumn{3}{|c|}{$\Omega / \Omega_{K} p_{c}\left(\mathrm{dyn} / \mathrm{cm}^{2}\right) \rho_{c}(\mathrm{~g})$} & \multirow{2}{*}{$\begin{array}{l}T_{c}(\mathrm{~K}) \\
2.43 \cdot 10^{7}\end{array}$} & \multirow{2}{*}{$\begin{array}{l}p_{s} / p_{c} \\
9.61 \cdot 10^{-15}\end{array}$} & \multirow{2}{*}{$\begin{array}{l}\rho_{s} / \rho_{c} \\
2.03 \cdot 10^{-11}\end{array}$} & \multirow{2}{*}{$\begin{array}{l}\begin{array}{l}\text { Virial } \\
\text { test }\end{array} \\
5.08 \cdot 10^{-11}\end{array}$} & \multirow{2}{*}{$\begin{array}{l}\begin{array}{l}\text { Energy } \\
\text { test }{ }^{c}\end{array} \\
5.84 \cdot 10^{-7}\end{array}$} \\
\hline & 0.0 & $1.34 \cdot 10^{17}$ & 40.8 & & & & & \\
\hline 3.0 & 0.3 & $1.34 \cdot 10^{17}$ & 41.0 & $2.43 \cdot 10^{7}$ & $9.92 \cdot 10^{-15}$ & $2.07 \cdot 10^{-11}$ & $8.01 \cdot 10^{-11}$ & $3.42 \cdot 10^{-7}$ \\
\hline 3.0 & 0.5 & $1.35 \cdot 10^{17}$ & 41.3 & $2.42 \cdot 10^{7}$ & $1.03 \cdot 10^{-14}$ & $2.09 \cdot 10^{-11}$ & $2.03 \cdot 10^{-10}$ & $6.14 \cdot 10^{-6}$ \\
\hline 3.0 & 0.7 & $1.35 \cdot 10^{17}$ & 41.5 & $2.42 \cdot 10^{7}$ & $1.07 \cdot 10^{-14}$ & $2.08 \cdot 10^{-11}$ & $2.67 \cdot 10^{-10}$ & $7.50 \cdot 10^{-6}$ \\
\hline 3.0 & 0.9 & $1.36 \cdot 10^{17}$ & 41.6 & $2.42 \cdot 10^{7}$ & $1.09 \cdot 10^{-14}$ & $1.93 \cdot 10^{-11}$ & $2.31 \cdot 10^{-10}$ & $1.09 \cdot 10^{-5}$ \\
\hline 5.0 & 0.0 & & 21.3 & $2.76 \cdot 10^{7}$ & $3.86 \cdot 10^{-14}$ & $6.22 \cdot 10^{-11}$ & & \\
\hline 5.0 & 0.3 & $8.04 \cdot 10^{16}$ & 21.4 & $2.75 \cdot 10^{7}$ & $3.97 \cdot 10^{-14}$ & $6.30 \cdot 10^{-11}$ & $8.41 \cdot 10^{-11}$ & $8.84 \cdot 10^{-6}$ \\
\hline 5.0 & 0.5 & $8.09 \cdot 10^{16}$ & 21.6 & $2.75 \cdot 10^{7}$ & $4.11 \cdot 10^{-14}$ & $6.34 \cdot 10^{-11}$ & $7.51 \cdot 10^{-11}$ & \\
\hline 5.0 & 0.7 & $8.13 \cdot 10^{16}$ & 21.7 & $2.75 \cdot 10^{7}$ & $4.23 \cdot 10^{-14}$ & $6.21 \cdot 10^{-11}$ & $8.01 \cdot 10^{-11}$ & $7.33 \cdot 10^{-6}$ \\
\hline 5.0 & 0.9 & $8.16 \cdot 10^{16}$ & 21.8 & $2.74 \cdot 10^{7}$ & $4.29 \cdot 10^{-14}$ & $5.58 \cdot 10^{-11}$ & $1.06 \cdot 10^{-10}$ & $1.34 \cdot 10^{-5}$ \\
\hline 10.0 & 0.0 & $4.31 \cdot 10^{10}$ & 44 & $3.20 \cdot 10^{7}$ & $1.53 \cdot 10^{-13}$ & $1.79 \cdot 10^{-10}$ & $8.96 \cdot 10^{-12}$ & $1.12 \cdot 10$ \\
\hline 10.0 & 0.3 & $4.33 \cdot 10^{16}$ & 9.50 & $3.20 \cdot 10^{7}$ & $1.56 \cdot 10^{-13}$ & $1.80 \cdot 10^{-10}$ & $4.91 \cdot 10^{-11}$ & $5.53 \cdot 10^{-7}$ \\
\hline 10.0 & 0.5 & $4.35 \cdot 10^{16}$ & 9.58 & $3.19 \cdot 10^{7}$ & $1.60 \cdot 10^{-13}$ & $1.78 \cdot 10^{-10}$ & $5.18 \cdot 10^{-11}$ & $1.25 \cdot 10^{-6}$ \\
\hline 10.0 & 0.7 & $4.38 \cdot 10^{16}$ & 9.65 & $3.19 \cdot 10^{7}$ & $1.64 \cdot 10^{-13}$ & $1.69 \cdot 10^{-10}$ & $2.59 \cdot 10^{-11}$ & $5.62 \cdot 10^{-7}$ \\
\hline 10.0 & 0.9 & $4.39 \cdot 10^{16}$ & 9.69 & $3.18 \cdot 10^{7}$ & $1.66 \cdot 10^{-13}$ & $1.34 \cdot 10^{-10}$ & $4.54 \cdot 10^{-11}$ & $4.48 \cdot 10^{-7}$ \\
\hline 0.0 & 0 & & 。 & $3.62 \cdot 10^{7}$ & $3.26 \cdot 10^{-13}$ & $2.41 \cdot 10^{-10}$ & $1.54 \cdot 10^{-11}$ & \\
\hline 20.0 & 0.3 & $2.80 \cdot 10^{16}$ & 4.91 & $3.61 \cdot 10^{7}$ & $3.31 \cdot 10^{-13}$ & $2.36 \cdot 10^{-10}$ & $7.25 \cdot 10^{-11}$ & $1.49 \cdot 10^{-6}$ \\
\hline 20.0 & 0.5 & $2.81 \cdot 10^{16}$ & 4.94 & $3.61 \cdot 10^{7}$ & $3.39 \cdot 10^{-13}$ & $2.23 \cdot 10^{-10}$ & $1.79 \cdot 10^{-11}$ & $2.11 \cdot 10^{-7}$ \\
\hline 20.0 & 0.7 & $2.82 \cdot 10^{16}$ & 4.98 & $3.60 \cdot 10^{7}$ & $3.46 \cdot 10^{-13}$ & $1.93 \cdot 10^{-10}$ & $7.16 \cdot 10^{-11}$ & $4.38 \cdot 10^{-8}$ \\
\hline 20.0 & 0.9 & $2.83 \cdot 10^{16}$ & 5.00 & $3.60 \cdot 10^{7}$ & $3.49 \cdot 10^{-13}$ & $1.17 \cdot 10^{-10}$ & $4.09 \cdot 10^{-11}$ & $1.09 \cdot 10^{-6}$ \\
\hline
\end{tabular}

${ }^{a}$ From left to right: Mass, equatorial angular velocity, central pressure, central density, central temperature, ratio between surface and central pressure, ratio between surface and central density, relative virial test and relative energy test. The values for the solar parameters used in the table are $M_{\odot}=1.9891 \cdot 10^{33} \mathrm{~g}, R_{\odot}=6.95508 \cdot 10^{10} \mathrm{~cm}$ and $L_{\odot}=3.8396 \cdot 10^{33} \mathrm{erg} / \mathrm{s}$.

${ }^{b}$ Virial test: $\left(2 T_{\text {rel }}+I \Omega_{\star}^{2}+W+3 P+I_{s}+2 \Omega_{\star} L_{z}\right) / W$.

${ }^{c}$ Energy test: $\left(\int_{(S)}(\xi \nabla T) \cdot \mathrm{d} \mathbf{S}+\int_{(V)} \varepsilon \mathrm{d} V\right) / \int_{(V)} \varepsilon \mathrm{d} V$

- dynamical evolution during main sequence with stellar wind, which forces a spin down and associated mixing;

- nuclear evolution.

These steps need the right algorithm for temporal evolution, which is not known presently. Indeed, such an algorithm should be at the same time fast, stable and precise. We have managed to use spectral methods, which ensure rapidity and precision but the stability remains a challenge. A better understanding of the properties of the discretized operators is certainly a key to improve the efficiency of the algorithms. 
As shown by the foregoing examples, some realistic models can now be computed for intermediate mass and massive stars. These models are steady and therefore the chemical composition must be given. To circumvent this constraint, one-dimensional models can be used to compute time evolution and the ensuing chemical composition. Then, the bulk relation between pressure and chemical composition shown by the $1 \mathrm{D}$-model can be inserted into the 2D-model. In this way, steady models can include some stellar evolution.

The steady models described in this work are most relevant in the study of the oscillation properties of rotating stars. The interpretation of the frequency spectrum of such stars is indeed a challenging problem and an intense use of $2 \mathrm{D}$-models will be necessary to find out how to invert data coming from, for instance, $\delta$-Scuti stars.

Another use of these models is obviously the interpretation of interferometric data collected on some nearby fast rotating stars ( $\alpha$ Aql, $\alpha$ Cep, $\alpha$ Leo, $\beta$ Cas, etc.). Fast rotating stars have a surface brightness that strongly depends on latitude (gravity darkening). Accurate models are crucial to extract the physical parameters contained in the interferometric data from such stars.

Finally, steady two-dimensional models may also serve as proxies for the initial conditions of a collapsing massive star, although the final word will come from time-evolved models including mass-loss.

Acknowledgements The authors acknowledge the support of the French Agence Nationale de la Recherche (ANR), under grant ESTER (ANR-09-BLAN-0140). This work was also supported by the Centre National de la Recherche Scientifique (CNRS, UMR 5277), through the Programme National de Physique Stellaire (PNPS). The numerical calculations were carried out on the CalMip machine of the "Centre Interuniversitaire de Calcul de Toulouse" (CICT) which is gratefully acknowledged.

\section{References}

Bodenheimer, P. 1971, ApJ, 167, 153

Bodenheimer, P. \& Ostriker, J. P. 1970, ApJ, 161, 1101

Bodenheimer, P. \& Ostriker, J. P. 1973, ApJ, 180, 159

Bonazzola, S., Gourgoulhon, E., \& Marck, J.-A. 1998, Phys. Rev. D, 58, 104020

Canuto, C., Hussaini, M. Y., Quarteroni, A., \& Zang, T. A. 2006, Spectral Methods: Fundamentals in Single Domains (Springer Verlag)

Charbonnel, C. \& Talon, S. 1999, A\&A, 351, 635

Clement, M. J. 1974, ApJ, 194, 709

Deupree, R. G. 2011, ApJ, 735, 69

Dintrans, B. \& Rieutord, M. 2000, A\&A, 354, 86

Domiciano de Souza, A., Kervella, P., Jankov, S., et al. 2005, A\&A, 442, 567

Eriguchi, Y. 1978, Pub. Astron. Soc. Jap., 30, 507

Eriguchi, Y. \& Müller, E. 1985, A\&A, 146, 260 
Eriguchi, Y. \& Müller, E. 1991, A\&A, 248, 435

Eriguchi, Y. \& Sugimoto, D. 1981, Progress of Theoretical Physics, 65, 1870

Espinosa Lara, F. \& Rieutord, M. 2007, A\&A, 470, 1013

Espinosa Lara, F. \& Rieutord, M. 2011, A\&A, 533, A43

Espinosa Lara, F. \& Rieutord, M. 2012, in preparation

Fornberg, B. 1998, A practical guide to pseudospectral methods (Cambridge University Press)

Frischknecht, U., Hirschi, R., \& Thielemann, F.-K. 2012, A\&A, 538, L2

Grandclément, P. 2006, in Stellar fluid dynamics and numerical simulations: from the Sun to neutron stars, ed. M. Rieutord \& B. Dubrulle (EDP Sciences), $153-180$

Grevesse, N. \& Noels, A. 1993, in Origin and evolution of the elements, ed. N. Prantzos, E. Vangioni-Flam, \& M. Cassé (Cambridge Univ. Press), 1525

Jackson, S. 1970, ApJ, 161, 579

Jackson, S., MacGregor, K. B., \& Skumanich, A. 2004, ApJ, 606, 1196

Jackson, S., MacGregor, K. B., \& Skumanich, A. 2005, Astrophys. J. Supp. Ser., 156, 245

James, R. A. 1964, ApJ, 140, 552

Lovekin, C. C., Deupree, R. G., \& Short, C. I. 2006, ApJ, 643, 460

Maeder, A. \& Meynet, G. 2001, A\&A, 373, 555

Mark, J. W.-K. 1968, ApJ, 154, 627

Metzger, B. D., Giannios, D., Thompson, T. A., Bucciantini, N., \& Quataert, E. 2011, MNRAS, 413, 2031

Meynet, G. \& Maeder, A. 2005, A\&A, 429, 581

Ostriker, J. P. \& Bodenheimer, P. 1968, ApJ, 151, 1089

Ostriker, J. P. \& Hartwick, F. D. A. 1968, ApJ, 153, 797

Ostriker, J. P. \& Mark, J. W.-K. 1968, ApJ, 151, 1075

Reese, D. R., MacGregor, K. B., Jackson, S., Skumanich, A., \& Metcalfe, T. S. 2009a, A\&A, 506, 189

Reese, D. R., Thompson, M. J., MacGregor, K. B., et al. 2009b, A\&A, 506, 183

Rieutord, M. 2006a, A\&A, 451, 1025

Rieutord, M. 2006b, in SF2A proceeding 2006, ed. F. Casoli \& al., astro$\mathrm{ph} / 0702384$

Rieutord, M. \& Espinosa Lara, F. 2009, Comm. in Asteroseismology, 158, 99

Roxburgh, I. W. 2004, A\&A, 428, 171

Shindo, M., Hashimoto, M., Eriguchi, Y., \& Mueller, E. 1997, A\&A, 326, 177

Uryu, K. \& Eriguchi, Y. 1994, MNRAS, 269, 24

Uryu, K. \& Eriguchi, Y. 1995, MNRAS, 277, 1411

Zahn, J.-P. 1992, A\&A, 265, 115 